\title{
Antioxidant effect of lutein towards phospholipid hydroperoxidation in human erythrocytes
}

\author{
Kiyotaka Nakagawa ${ }^{1}$, Takehiro Kiko ${ }^{1}$, Keijiro Hatade ${ }^{1}$, Phumon Sookwong ${ }^{1}$, Hiroyuki Arai $^{2}$ \\ and Teruo Miyazawa ${ }^{1 *}$ \\ ${ }^{1}$ Food and Biodynamic Chemistry Laboratory, Graduate School of Agricultural Science, Tohoku University, Sendai 981-8555, \\ Japan \\ ${ }^{2}$ Department of Geriatrics and Gerontology, Tohoku University School of Medicine, Sendai 980-8574, Japan
}

(Received 12 November 2008 - Revised 21 January 2009 - Accepted 1 April 2009 - First published online 22 July 2009)

Peroxidised phospholipid-mediated cytotoxity is involved in the pathophysiology of many diseases; for example, phospholipid hydroperoxides $(\mathrm{PLOOH})$ are abnormally increased in erythrocytes of dementia patients. Dietary carotenoids (especially xanthophylls, polar carotenoids such as lutein) have gained attention as potent inhibitors against erythrocyte phospholipid hydroperoxidation, thereby making them plausible candidates for preventing diseases (i.e. dementia). To evaluate these points, we investigated whether orally administered lutein is distributed to human erythrocytes, and inhibits erythrocyte PLOOH formation. Six healthy subjects took one capsule of food-grade lutein ( $9.67 \mathrm{mg}$ lutein per capsule) once per d for 4 weeks. Before and during the supplementation period, carotenoids and PLOOH in erythrocytes and plasma were determined by our developed HPLC technique. The administered lutein was incorporated into human erythrocytes, and erythrocyte PLOOH level decreased after the ingestion for 2 and 4 weeks. The antioxidative effect of lutein was confirmed on erythrocyte membranes, but not in plasma. These results suggest that lutein has the potential to act as an important antioxidant molecule in erythrocytes, and it thereby may contribute to the prevention of dementia. Therefore future biological and clinical studies will be required to evaluate the efficacy as well as safety of lutein in models of dementia with a realistic prospect of its use in human therapy.

Lutein: Phospholipid hydroperoxides: Erythrocytes: Dementia

Peroxidation of membrane phospholipids (PL) has received attention in relation to oxidative stress occurring during pathophysiological changes such as atherogenesis, ageing and others $^{(1,2)}$. We previously confirmed that significantly higher levels of peroxidised PL (i.e. PL hydroperoxides; PLOOH) are accumulated in the erythrocytes of dementia patients ${ }^{(3)}$. Any compounds or food components that can minimise this accumulation could be used therapeutically as effective drugs or as functional foods to prevent the diseases. We therefore carried out animal studies and found that carotenoids were potential compounds to inhibit erythrocyte PL hydroperoxidation ${ }^{(4,5)}$.

From these circumstances, we are particularly interested in the effects of carotenoids on human erythrocytes, but information is very limited. In contrast, plasma carotenoids have been investigated thoroughly for analytical methods as well as biological metabolism and their significance. To the best of our knowledge, occurrence and roles of carotenoids in human erythrocytes have not been fully understood, and this has been due, in part, to a lack of the suitable analytical method.

To address this need, we recently developed a method for determining human erythrocyte carotenoids by using
HPLC $^{(6)}$. By using the method, evidence is accumulating that erythrocyte carotenoids (especially xanthophylls, polar carotenoids such as lutein) decrease, and erythrocyte $\mathrm{PLOOH}$ increase, in correlation with the severity of dementia. Erythrocytes with high levels of lipid hydroperoxides have been postulated to have a decreased ability to transport oxygen to the brain, and they may impair blood rheology ${ }^{(7,8)}$, thus facilitating dementia. On the basis of these points, it was hypothesised that xanthophylls would have potential to act as important antioxidant molecules in erythrocytes, and they thereby might contribute to the prevention of dementia.

To evaluate the hypothesis, we investigated whether administered lutein is distributed to human erythrocytes, and inhibits erythrocyte PL hydroperoxidation. To date, although there have been many reports about the health benefits of xanthophylls $^{(9)}$, they have never provided any information about the distribution and the effect of xanthophylls in erythrocytes. Therefore, our findings (the inhibitory effect of lutein on erythrocyte PLOOH) would provide a new insight into the application of lutein possibly as an anti-dementia agent.

Abbreviations: CL, chemiluminescence; DAD, diode array detection; PCOOH, phosphatidylcholine hydroperoxide; PEOOH, phosphatidylethanolamine

hydroperoxide; PL, phospholipid; PLOOH, phospholipid hydroperoxide.

* Corresponding author: Dr Teruo Miyazawa, fax +8122717 8905, email miyazawa@biochem.tohoku.ac.jp 


\section{Subjects and methods}

\section{Supplementation study}

Six healthy subjects (aged 21-28 years) participated in the present study. The subjects gave written informed consent to the experimental protocol. Subjects took one capsule of Flora-Glo Lutein (containing $9.67 \mathrm{mg}$ lutein, $0.73 \mathrm{mg}$ zeaxanthin and $0.12 \mathrm{mg} \alpha$-tocopherol; Kemin Foods, Des Moines, IA, USA) once per d (after breakfast) for 4 weeks. During the experimental period, the subjects were instructed to avoid foods rich in carotenoids (for example, spinach, broccoli, carrots and tomatoes). Before, 2 and 4 weeks after the ingestion, blood was collected into a tube containing EDTA-2Na as an anticoagulant. The blood was subjected to centrifugation at $1000 \mathrm{~g}$ for $10 \mathrm{~min}$ at $4^{\circ} \mathrm{C}$. After the plasma and buffy coat were removed, erythrocytes were washed three times with PBS ( $\mathrm{pH} 7.4)$ to prepare packed cells. The packed cells were immediately subjected to determination of carotenoids and PLOOH. Plasma samples were stored at $-80^{\circ} \mathrm{C}$ until analysis.

\section{Measurement of carotenoids in erythrocytes}

For determination of erythrocyte carotenoids $^{(6)}$, packed cells $(2.5 \mathrm{ml})$ were diluted with $2.5 \mathrm{ml}$ of water, and were mixed with $5 \mathrm{ml}$ of $80 \mathrm{~mm}$-ethanolic pyrogallol, $1.0 \mathrm{ml}$ of $1.8 \mathrm{M}$-aqueous $\mathrm{KOH}$ and $40 \mu \mathrm{l}$ of $1 \mu \mathrm{M}$-ethanolic echinenone (internal standard). After addition of $1.25 \mathrm{ml}$ of $0.1 \mathrm{M}$-aqueous sodium dodecyl sulfate, the sample was mixed with $15 \mathrm{ml}$ of hexane-dichloromethane $(5: 1, \mathrm{v} / \mathrm{v})$ for extraction of erythrocyte carotenoids. The extract was purified by Sep-Pak silica cartridge (Waters, Milford, MA, USA), and then subjected to HPLC coupled with UV diode array detection (DAD) and atmospheric pressure chemical ionisation MS. A C30 Carotenoid column $(4.6 \times 250 \mathrm{~mm}, 5 \mu \mathrm{m}$; YMC, Kyoto, Japan) was used. The column was eluted using a binary gradient consisting of the following HPLC solvents: A, methanoltert-butyl ether (MTBE)-water (83:15:2, by vol.; containing $3.9 \mathrm{mM}$-ammonium acetate); B, methanol-MTBE-water (8:90:2, by vol.; containing $2.6 \mathrm{~mm}$-ammonium acetate). The gradient profile was as follows: $0-12 \mathrm{~min}, 10-45 \% \mathrm{~B}$ linear; $12-24 \mathrm{~min}, 45-100 \% \mathrm{~B}$ linear; $24-30 \mathrm{~min}, 100 \%$
B. The flow rate was adjusted to $1 \mathrm{ml} / \mathrm{min}$, and the column temperature was maintained at $20^{\circ} \mathrm{C}$. The column eluent was sent to a Shimadzu SPD-M10Avp DAD detector (Kyoto, Japan) for monitoring carotenoids at $463 \mathrm{~nm}$. Concentrations of erythrocyte carotenoids were calculated using the equation corresponding to the external standard curve of each carotenoid and were adjusted by the percentage recovery of the added echinenone (internal standard).

\section{Measurement of phospholipid hydroperoxides in erythrocytes}

For determination of erythrocyte $\mathrm{PLOOH}^{(2-5)}$, total lipids were extracted from packed cells with a mixture of 2-propanol and chloroform containing butylated hydroxytoluene. PLOOH (i.e. phosphatidylcholine hydroperoxide $(\mathrm{PCOOH})$ and phosphatidylethanolamine hydroperoxide $(\mathrm{PEOOH}))$ in the total lipids were measured by HPLC with chemiluminescence (CL) detection. The column was a $4.6 \times 250 \mathrm{~mm}, 5 \mu \mathrm{m}$ Finepak SIL NH2-5 (Japan Spectroscopic Co., Tokyo, Japan), the eluent was 2-propanol-methanol-water (135:45:20, by vol.) and the flow rate was $1.0 \mathrm{ml} / \mathrm{min}$. Post-column CL detection was carried out using a CLD-100 detector (Tohoku Electronic Industries Co., Sendai, Japan). A mixture of luminol and cytochrome $\mathrm{c}$ in $50 \mathrm{~mm}$-borate buffer $(\mathrm{pH} 10 \cdot 0)$ was used as a hydroperoxide-specific post-column CL reagent. Calibration was carried out using standard $\mathrm{PCOOH}$ or $\mathrm{PEOOH}$.

\section{Other analytical methods}

For plasma samples, carotenoids and PLOOH were determined by HPLC-UV ${ }^{(4)}$ and HPLC-CL ${ }^{(2-5)}$, respectively. Tocopherols in erythrocytes and plasma were measured by HPLC with fluorescence detection ${ }^{(10)}$. Erythrocyte and plasma PL were quantified by Bartlett's method ${ }^{(11)}$. Erythrocyte $\mathrm{Hb}$ was measured by a commercial kits (Wako, Osaka, Japan).

\section{Statistical analyses}

Data were expressed as mean values and standard deviations. Statistical analysis was performed by ANOVA followed by Tukey's test. Differences were considered significant at $P<0.05$.
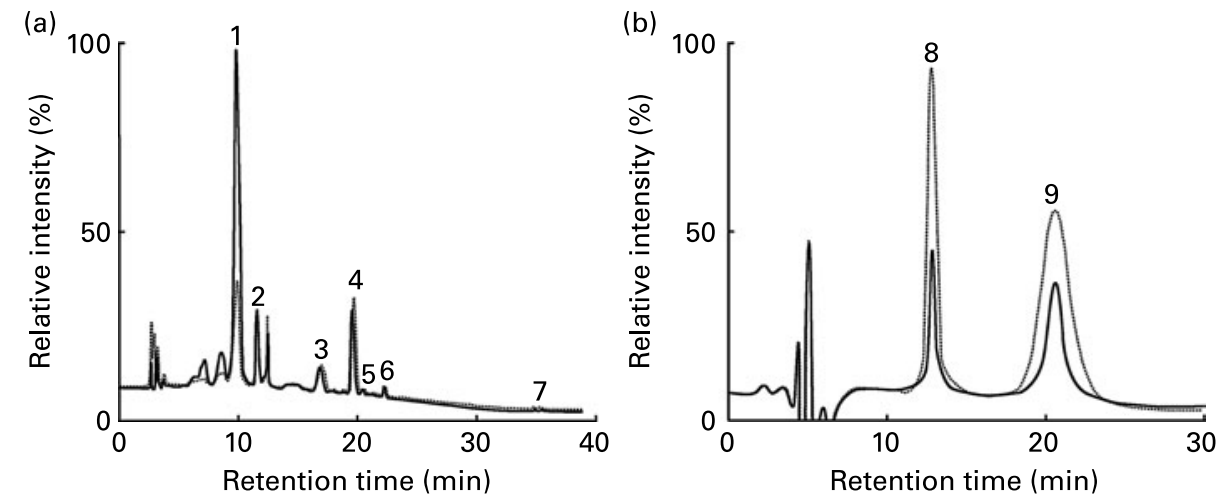

Fig. 1. Typical diode array detection (DAD) and chemiluminescence (CL) chromatograms of carotenoids (a) and phospholipid hydroperoxides (PLOOH) (b) in erythrocytes taken before (…) and 4 weeks after $(-)$ ingestion of food-grade lutein (Flora-Glo Lutein; Kemin Foods, Des Moines, IA, USA). Erythrocyte carotenoids and PLOOH were determined by HPLC-DAD and HPLC-CL, respectively. Peak identifications are as follows: 1 , lutein; 2 , zeaxanthin; $3, \beta$-cryptoxanthin; 4, echinenone (internal standard); $5, \alpha$-carotene; $6, \beta$-carotene; 7, lycopene; 8, phosphatidylcholine hydroperoxide; 9, phosphatidylethanolamine hydroperoxide. 


\section{Results}

In a typical DAD chromatogram of erythrocyte extract taken before the ingestion of Flora-Glo Lutein, six endogenous carotenoids (lutein, zeaxanthin, $\beta$-cryptoxanthin, $\alpha$-carotene, $\beta$-carotene and lycopene) were separated, detected by DAD (Fig. 1 (a)), and concurrently identified based on atmospheric pressure chemical ionisation MS and UV spectra profiles (data not shown). The relative amounts of each carotenoid were consistently as follows: lutein $>\beta$-cryptoxanthin $>$ zeaxanthin $>\beta$-carotene $>$ lycopene $>\alpha$-carotene (Table 1 ). These data support our previous finding that, in human erythrocytes, xanthophylls (especially lutein) are the most prevalent carotenoids ${ }^{(6)}$. In contrast, non-polar carotenoids (for example, $\beta$-carotene) were more abundant in plasma (Table 1). In a typical CL chromatogram of erythrocyte total lipids taken before the ingestion, $\mathrm{PCOOH}$ and $\mathrm{PEOOH}$ were the predominant forms of PLOOH (Fig. 1 (b)). On the other hand, only $\mathrm{PCOOH}$ was detected in plasma (Table 1).

After ingestion of Flora-Glo Lutein for 4 weeks, erythrocyte lutein concentration increased from baseline (160 (SD 42) $\mathrm{pmol} / \mathrm{g} \mathrm{Hb}$ ) to 449 (SD 120) pmol/g Hb, whereas erythrocyte $\mathrm{PLOOH}$ (sum of $\mathrm{PCOOH}$ and $\mathrm{PEOOH}$ ) decreased from 4.9 (SD 2.5) $\mu \mathrm{mmol} / \mathrm{mol}$ PL to 1.5 (SD 1.2 ) $\mu \mathrm{mmol} / \mathrm{mol} \mathrm{PL}$ (Table 1). Flora-Glo Lutein ingestion did not affect the levels of zeaxanthin, $\beta$-carotene, lycopene and $\alpha$-carotene in erythrocytes and plasma, PLOOH in plasma, and tocopherols in erythrocytes and plasma (Table 1). Also, erythrocyte $\mathrm{Hb}$, erythrocyte PL as well as plasma PL were not affected by Flora-Glo Lutein ingestion (data not shown). Based on these results, it is suggested that when humans ingest lutein, lutein is absorbed, distributed and accumulated in erythrocytes, where it acts as an antioxidant molecule, thereby reducing $\mathrm{PLOOH}$ as an index of oxidative stress.

Table 1. Carotenoid, phospholipid hydroperoxide (PLOOH) and tocopherol contents in erythrocytes and plasma taken before and after ingestion of food-grade lutein (Flora-Glo Lutein $\left.{ }^{\star}\right)(n 6)$

(Mean values and standard deviations)

\begin{tabular}{|c|c|c|c|c|c|c|}
\hline & \multicolumn{2}{|c|}{ Before ingestion } & \multicolumn{2}{|c|}{2 weeks after ingestion } & \multicolumn{2}{|c|}{4 weeks after ingestion } \\
\hline & Mean & SD & Mean & SD & Mean & SD \\
\hline \multicolumn{7}{|l|}{ Erythrocytes } \\
\hline \multicolumn{7}{|l|}{ Carotenoids $(\mathrm{pmol} / \mathrm{g} \mathrm{Hb})$} \\
\hline Lutein & $160^{\mathrm{a}}$ & 42 & $364^{\mathrm{b}}$ & 113 & $449^{b}$ & 120 \\
\hline Zeaxanthin & 52 & 18 & 66 & 21 & 71 & 18 \\
\hline$\beta$-Cryptoxanthin & 66 & 19 & 64 & 17 & 68 & 21 \\
\hline$\alpha$-Carotene & 8 & 5 & 9 & 6 & 5 & 4 \\
\hline$\beta$-Carotene & 20 & 9 & 22 & 14 & 11 & 4 \\
\hline Lycopene & 12 & 6 & 14 & 10 & 5 & 2 \\
\hline Xanthophylls† & $278^{a}$ & 47 & $495^{\mathrm{b}}$ & 116 & $589^{b}$ & 118 \\
\hline Non-polar carotenoids $\ddagger$ & 40 & 18 & 46 & 28 & 21 & 10 \\
\hline Total carotenoids & $318^{\mathrm{a}}$ & 57 & $540^{\mathrm{b}}$ & 110 & $610^{\mathrm{b}}$ & 110 \\
\hline \multicolumn{7}{|l|}{ PLOOH ( $\mu \mathrm{mol} / \mathrm{mol}$ phospholipids) } \\
\hline $\mathrm{PCOOH}$ & $2 \cdot 7^{\mathrm{a}}$ & 1.4 & $1 \cdot 7^{\mathrm{a}}$ & 0.6 & $0.7^{\mathrm{b}}$ & 0.5 \\
\hline $\mathrm{PEOOH}$ & $2 \cdot 1^{\mathrm{a}}$ & $1 \cdot 1$ & $0.7^{\mathrm{b}}$ & 0.2 & $0.8^{\mathrm{b}}$ & 0.8 \\
\hline Total PLOOH (PCOOH + PEOOH) & $4 \cdot 9^{\mathrm{a}}$ & 2.5 & $2 \cdot 4^{\mathrm{a}}$ & 0.8 & $1.5^{\mathrm{b}}$ & $1 \cdot 2$ \\
\hline \multicolumn{7}{|l|}{ Tocopherols $(\mathrm{nmol} / \mathrm{g} \mathrm{Hb})$} \\
\hline$\alpha$-Tocopherol & $20 \cdot 2$ & $3 \cdot 1$ & $21 \cdot 2$ & $2 \cdot 9$ & $20 \cdot 7$ & 0.8 \\
\hline$\gamma$-Tocopherol & 3.0 & 0.6 & $3 \cdot 8$ & $1 \cdot 1$ & $3 \cdot 2$ & 0.5 \\
\hline Total tocopherols & 23.2 & 3.0 & $25 \cdot 0$ & $3 \cdot 2$ & 23.9 & 0.9 \\
\hline \multicolumn{7}{|l|}{ Plasma } \\
\hline \multicolumn{7}{|l|}{ Carotenoids (pmol/ml) } \\
\hline Lutein & $341^{\mathrm{a}}$ & 119 & $682^{a, b}$ & 280 & $768^{\mathrm{b}}$ & 295 \\
\hline Zeaxanthin & 115 & 33 & 122 & 46 & 120 & 38 \\
\hline$\beta$-Cryptoxanthin & $411^{\mathrm{b}}$ & 111 & $284^{\mathrm{a}}$ & 77 & $224^{a}$ & 31 \\
\hline$\alpha$-Carotene & 184 & 94 & 147 & 56 & 135 & 48 \\
\hline$\beta$-Carotene & 475 & 189 & 409 & 168 & 337 & 175 \\
\hline Lycopene & 303 & 140 & 249 & 119 & 301 & 112 \\
\hline Xanthophylls $\dagger$ & 868 & 151 & 1083 & 361 & 1112 & 317 \\
\hline Non-polar carotenoids $\ddagger$ & 961 & 377 & 805 & 303 & 772 & 255 \\
\hline Total carotenoids & 1829 & 452 & 1894 & 445 & 1885 & 279 \\
\hline \multicolumn{7}{|l|}{ PLOOH ( $\mu \mathrm{mol} / \mathrm{mol}$ phospholipids) } \\
\hline $\mathrm{PCOOH}$ & 9.5 & $5 \cdot 1$ & $7 \cdot 7$ & $2 \cdot 2$ & $11 \cdot 3$ & 3.9 \\
\hline \multicolumn{7}{|l|}{ Tocopherols $(\mathrm{nmol} / \mathrm{ml})$} \\
\hline$\alpha$-Tocopherol & $24 \cdot 4$ & $4 \cdot 3$ & $22 \cdot 7$ & $4 \cdot 0$ & $23 \cdot 7$ & $3 \cdot 6$ \\
\hline$\gamma$-Tocopherol & $2 \cdot 7$ & 0.6 & $3 \cdot 1$ & 0.5 & $3 \cdot 0$ & 0.4 \\
\hline Total tocopherols & $27 \cdot 1$ & 4.6 & $25 \cdot 7$ & $3 \cdot 6$ & $26 \cdot 6$ & $3 \cdot 8$ \\
\hline
\end{tabular}

$\mathrm{PCOOH}$, phosphatidylcholine hydroperoxide; $\mathrm{PEOOH}$, phosphatidylethanolamine hydroperoxide.

a,b,c Mean values within a column with unlike superscript letters were significantly different $(P<0.05)$.

${ }^{*}$ Kemin Foods (Des Moines, IA, USA).

$\dagger$ Xanthophylls are the sum of lutein, zeaxanthin and $\beta$-cryptoxanthin.

$\ddagger$ Non-polar carotenoids are the sum of $\alpha$-carotene, $\beta$-carotene and lycopene. 


\section{Discussion}

In recent years, the antioxidative property of food constituents has been seriously noted by medical and nutritional experts, since the reactive oxygen species-mediated peroxidation of biological molecules (for example, lipids) has been postulated to induce a variety of pathological events such as atherogenesis, ageing and dementia. Although many in vitro studies on the antioxidative property of food constituents have been reported, little has been known about the biological functions of dietary antioxidants in vivo (especially in humans), except for major antioxidants (for example, tocopherols and ascorbic acid). Since the bioavailability of food constituents is limited by their digestibility and metabolic fate, oral administration trials are favoured to evaluate their biological function.

The present study demonstrates that when human subjects ingest food-grade lutein (Flora-Glo Lutein), lutein is absorbed, distributed and accumulated in erythrocytes, where it exhibits antioxidative effects in vivo (inhibition of erythrocyte PL hydroperoxidation). It is interesting that the antioxidative effect observed in the present study was brought about by relatively short-term supplementation with lutein $(2-4$ weeks). In the present study, each volunteer received $9.67 \mathrm{mg}$ lutein per d. The dosage was about 2-fold compared with that of human daily intake ${ }^{(12)}$.

Until now, there have been about ten reports concerning human erythrocyte carotenoids. Some studies successfully detected erythrocyte carotenoids (mainly $\beta$-carotene) ${ }^{(13)}$, while the others were unable to detect them ${ }^{(14)}$. Incorporation of carotenoid ( $\beta$-carotene) into erythrocytes after oral supplementation was described in some reports ${ }^{(15)}$. However, there has been no study evaluating whether administered carotenoids other than $\beta$-carotene are distributed to the erythrocytes. In the present study, incorporation of lutein into erythrocytes after oral supplementation was well documented (Fig. 1 (a)). Because erythrocyte lutein concentration increased along with the elevation of plasma lutein (Table 1), it seems likely that lutein in plasma lipoprotein particles is partly transferred into erythrocytes. By this hypothesis, lutein would be located on the outer region of plasma lipoprotein, which facilitates its transfer to erythrocytes. On the other hand, the concentrations of endogenous antioxidants (i.e. carotenoids and tocopherols) other than erythrocyte and plasma lutein and plasma $\beta$-cryptoxanthin showed no changes before and after Flora-Glo Lutein ingestion (Table 1). This is advantageous for elucidation of the antioxidative contribution of lutein. According to the decrease of plasma $\beta$-cryptoxanthin (Table 1), this seems to be because the volunteers did not ingest foods enriched in $\beta$-cryptoxanthin throughout the supplementation period.

In the present study, to evaluate the peroxidisability, we measured PLOOH. Because PLOOH are the primary oxidation products of PL, an increase in PLOOH directly reflects in vivo oxidative stress ${ }^{(16)}$. As evidenced here, lutein supplementation clearly reduced erythrocyte PLOOH concentration (Fig. 1 (b)), indicating that lutein incorporated into erythrocytes attenuated PL peroxidation of erythrocyte membranes. On the other hand, the antioxidative effect of lutein was confirmed on the erythrocyte membrane, but not in the plasma (Table 1). Erythrocytes are rich in PUFA in the PL bilayer, and contain high concentrations of molecular oxygen and ferrous ions as constituents of oxyhaemoglobin $\mathrm{Hb}$. The oxidation of $\mathrm{Hb}$ accompanies the formation of superoxides, the source of more reactive oxygen species. Therefore, erythrocyte membrane PL would be more susceptible to peroxidation than other organelle membranes, although being protected by several antioxidative systems such as superoxide dismutase, catalase and glutathione peroxidase.

Recently, polar carotenoids (xanthophylls) have gained increasing scientific interest due to their antioxidative, antiobesity and anti-inflammatory activities ${ }^{(17)}$, which differ somewhat from those of non-polar carotenoids (for example, $\beta$-carotene). As mentioned in the Introduction, we previously found that the higher accumulation of PLOOH in the erythrocytes of dementia patients ${ }^{(3)}$ and the inhibitory effect on PLOOH formation in erythrocyte membranes was confirmed in mice by dietary supplementation with carotenoids ${ }^{(4,5)}$. In another study, a pronounced distribution of xanthophylls (for example, lutein) to erythrocytes was confirmed ${ }^{(6)}$. In the present study, orally administered lutein was incorporated into erythrocytes, and erythrocyte PLOOH levels decreased. This was supported by our ongoing study, in which human volunteers daily received chlorella rich in lutein. According to another ongoing study, erythrocyte PLOOH increased, and erythrocyte xanthophylls (for example, lutein) decreased, in correlation with the severity of dementia. Erythrocytes high in lipid hydroperoxides have been suggested to have a decreased ability to transport oxygen to brain, and they may impair blood rheology $y^{(7,8)}$, thus facilitating dementia. On the basis of these points, it seems that xanthophylls, especially lutein, have the potential to act as important antioxidant molecules in erythrocytes, and they thereby may contribute to the prevention of dementia. This possibility warrants their testing in other models of dementia with a realistic prospect of their use in human therapy.

\section{Acknowledgements}

Financial support was provided by Project M Co. Ltd (Sendai, Japan). The present study was also supported by Grants-in-Aid for Scientific Research (KAKENHI; 20228002) of the Japanese Society of the Promotion of Science (JSPS; Tokyo, Japan).

K. N. was involved in data collection, data analysis, data interpretation, literature search and manuscript preparation. T. K., K. H. and P. S. were involved in data collection, data analysis and data interpretation. H. A. and T. M. were involved in study design, data interpretation and review of the manuscript.

None of the authors has conflicts of interest with respect to the present study.

\section{References}

1. Stocker R \& Keaney JF Jr (2004) Role of oxidative modifications in atherosclerosis. Physiol Rev 84, 1381-1478.

2. Miyazawa T, Suzuki T, Fujimoto K, et al. (1992) Chemiluminescent simultaneous determination of phosphatidylcholine hydroperoxide and phosphatidylethanolamine hydroperoxide in the liver and brain of the rat. J Lipid Res 33, 1051-1059.

3. Miyazawa T, Suzuki T, Yasuda K, et al. (1992) Accumulation of phospholipid hydroperoxides in red blood cell membranes 
in Alzheimer disease. In Oxygen Radicals, pp. 327-330 [K Yagi, $\mathrm{M}$ Kondo, $\mathrm{E}$ Niki and $\mathrm{T}$ Yoshikawa, editors]. Amsterdam: Elsevier Science Publishing.

4. Nakagawa K, Fujimoto K \& Miyazawa T (1996) $\beta$-Carotene as a high-potency antioxidant to prevent the formation of phospholipid hydroperoxides in red blood cells of mice. Biochim Biophys Acta 1299, 110-116.

5. Asai A, Nakagawa K \& Miyazawa T (1999) Antioxidative effects of turmeric, rosemary and capsicum extracts on membrane phospholipid peroxidation and liver lipid metabolism in mice. Biosci Biotechnol Biochem 63, 2118-2122.

6. Nakagawa K, Kiko T, Hatade K, et al. (2008) Development of an HPLC-based assay for carotenoids in human red blood cells: application to clinical studies. Anal Biochem 381, 129-134.

7. Solerte SB, Ceresini G, Ferrari E, et al. (2000) Hemorheological changes and overproduction of cytokines from immune cells in mild to moderate dementia of the Alzheimer's type: adverse effects on cerebromicrovascular system. Neurobiol Aging 21, 271-281.

8. Ajmani RS, Metter EJ, Jaykumar R, et al. (2000) Hemodynamic changes during aging associated with cerebral blood flow and impaired cognitive function. Neurobiol Aging 21, 257-269.

9. Ribaya-Mercado JD \& Blumberg JB (2004) Lutein and zeaxanthin and their potential roles in disease prevention. J Am Coll Nutr 23, 567S-587S
10. Ikeda S, Tohyama T, Yoshimura H, et al. (2003) Dietary $\alpha$-tocopherol decreases $\alpha$-tocotrienol but not $\gamma$-tocotrienol concentration in rats. J Nutr 133, 428-434.

11. Bartlett GR (1959) Phosphorus assay in column chromatography. J Biol Chem 234, 466-468.

12. Kita J, Tada J, Ito $\mathbf{M}$, et al. (2004) Intake of phytochemicals among Japanese, calculated by the new FFF database. Biofactors 22, 259-263.

13. Fotouhi N, Meydani M, Santos MS, et al. (1996) Carotenoid and tocopherol concentrations in plasma, peripheral blood mononuclear cells, and red blood cells after long-term $\beta$-carotene supplementation in men. Am J Clin Nutr 63, 553-558.

14. Norkus EP, Bhagavan HN \& Nair PP (1990) Relationship between individual carotenoids in plasma, platelets and red blood cells (RBC) of adult subjects. FASEB J 4, A1774.

15. Murata T, Tamai H, Morinobu T, et al. (1992) Determination of $\beta$-carotene in plasma, blood cells and buccal mucosa by electrochemical detection. Lipids 27, 840-843.

16. Miyazawa T, Yasuda K, Fujimoto K, et al. (1988) Presence of phosphatidylcholine hydroperoxide in human plasma. $J$ Biochem 103, 744-746.

17. Izumi-Nagai K, Nagai N, Ohgami K, et al. (2007) Macular pigment lutein is antiinflammatory in preventing choroidal neovascularization. Arterioscler Thromb Vasc Biol 27, 2555-2562. 